\title{
Mechanism of Electron-Beam Induced Au Formation in Aqueous Solution
}

\author{
Jeung Hun Park ${ }^{1}$, Joseph M. Grogan ${ }^{2}$, Haim H. Bau ${ }^{2}$, Suneel Kodambaka ${ }^{1}$ and Frances M. Ross ${ }^{3}$ \\ ${ }^{1}$ Department of Materials Science and Engineering, University of California Los Angeles, \\ 410 Westwood Plaza, Los Angeles, CA 90095 \\ ${ }^{2}$ Department of Mechanical Engineering and Applied Mechanics, University of Pennsylvania, \\ 220 South 33rd Street, Philadelphia, PA 19147 \\ ${ }^{3}$ IBM T. J. Watson Research Center, 1101 Kitchawan Road, Yorktown Heights, NY 10598
}

Liquid cell-based transmission electron microscopy (TEM) enables the observation of structural changes and chemical processes in liquid phases. It provides a combination of temporal and spatial resolution that is difficult to achieve using other characterization techniques [1-3], and has yielded detailed information on solution-phase growth kinetics $[3,4]$. However, the electron beam can play a critical role in such experiments [4-10]. A quantitative understanding of the underlying physical mechanisms of electron beam effects, such as radiolysis, is essential for correct interpretation of growth measurements.

Here, we apply a simplified reaction-diffusion model to quantify the effect of beam induced radiolysis on nanoparticle growth. We examined synthesis of $\mathrm{Au}$ nanoparticles in a commonly used solution, $\mathrm{HAuCl}_{4}$. Experiments were carried out in a nanofluidic liquid-cell TEM device, the "nanoaquarium" [11, 12], in a Hitachi H9000 TEM operated at $300 \mathrm{keV}[2,3,10]$. Reduction of the soluble metallic precurs or, $\mathrm{Au}^{3+}$, results in the precipitation of $\mathrm{Au}$ as nanoparticles. Beam induced radiolysis of water generates radicals and molecular species such as $e_{h}^{-}, \mathrm{H}^{*}, \mathrm{OH}^{*}, \mathrm{H}_{2}, \mathrm{H}_{2} \mathrm{O}_{2}, \mathrm{H}_{3} \mathrm{O}^{+}$[9]. Many of these species, such as hydrated electrons, hydrogen radicals, and hydroxide radicals, can act as reducing agents and initiate the $\mathrm{Au}^{3+}$ reduction.

Figure 1 presents an example of the nucleation and growth of $\mathrm{Au}$ on the liquid cell windows. The projected area of $\mathrm{Au}$ crystallites is plotted as a function of time in Figure 2. We identify three distinct regimes. At the earliest times $(t<10 \mathrm{sec})$, nucleation occurs. For intermediate times $(10<t<145 \mathrm{sec})$, the area increases linearly with time. For later times $(145<t<320 \mathrm{sec})$, coalescence occurs and the growth rate decreases. Growth rate measurements on individual particles [10], where the thickness of each particle can be determined, suggest that the volume of Au (as well as the projected area) increases linearly with time at intermediate times. We measure this linear deposition rate as a function of beam current, total dose, and liquid thickness.

To quantify the growth observations, we developed a simplified reaction-diffusion model that incorporates the formation rate of radiolysis species, the reverse reactions that remove these species from solution, and the diffusion of each species out of the irradiated region [13]. By adding to the model the diffusive transport and attachment of $\mathrm{Au}$ at the growing interface, we can derive the growth rate for individual $\mathrm{Au}$ particles. A comparison of theoretical predictions with the experimental observations, particularly for different beam fluxes, allows us to understand the concentration distribution of the radiolytic species. We find that the beam induced growth of Au depends not only on the rate of diffusion of $\mathrm{Au}$ ions but also on the production and diffusion of the radiolytic species, allowing parameters of the radiolysis species to be investigated. The experimental and theoretical approaches presented here are general and likely to be applicable to addressing the question of beam effects in other liquid cell growth experiments. 
References:

[1] N. de Jonge and F. M. Ross, Nature Nanotechnology 6 (2011), 695.

[2] M. J. Williamson, R. M. Tromp, P. M. Vereecken, R. Hull, and F. M. Ross, Nature Materials 2 (2003), 532.

[3] A. Radisic, P. M. Vereecken, J. B. Hannon, P. C. Searson, and F. M. Ross, Nano Letters 6 (2006), 238.

[4] H. Zheng, R. K. Smith, Y.-W. Jun, C. Kisielowski, U. Dahmen, and A. P. Alivisatos, Science 324 (2009), 1309.

[5] J. E. Evans, K. L. Jungjohann, N. D. Browning, and I. Arslan, Nano Letters 11 (2011), 2809.

[6] H.-G. Liao, L. Cui, S. Whitelam, and H. Zheng, Science 336 (2012), 1011.

[7] J. M. Yuk, J. Park, P. Ercius, K. Kim, D. J. Hellebusch, M. F. Crommie, J. Y. Lee, A. Zettl, and A. P. Alivisatos, Science 336 (2012), 61.

[8] K. W. Noh, Y. Liu, L. Sun, and S. J. Dillon, Ultramicroscopy 116 (2012), 34.

[9] T. J. Woehl, J. E. Evans, I. Arslan, W. D. Ristenpart, and N. D. Browning, ACS Nano 6 (2012), 8599. [10] J. H. Park, J. M. Grogan, H. H. Bau, S. Kodambaka, and F. M. Ross, Microscopy and Microanalysis 18 Suppl. 2 (2012), 1098.

[11] J. Grogan and H. H. Bau, IEEE/ASME Journal of Microelectromechanical Systems 19 (2010), 885.

[12] J. M. Grogan, L. Rotkina, and H. H. Bau, Physical Review E 83 (2011), 061405.

[13] J. M. Grogan, F. M. Ross, and H. H. Bau, submitted (2013).

[14] We acknowledge the assistance of M. C. Reuter and A. W. Ellis at the IBM T.J. Watson Research

Center. This research was partially funded by the National Science Foundation under grants NSFGOALI (CMMI 1129722), NSF-NIRT (CBET 1066573) and NSF-NBIC (NSF NSECDMR-08-32802).

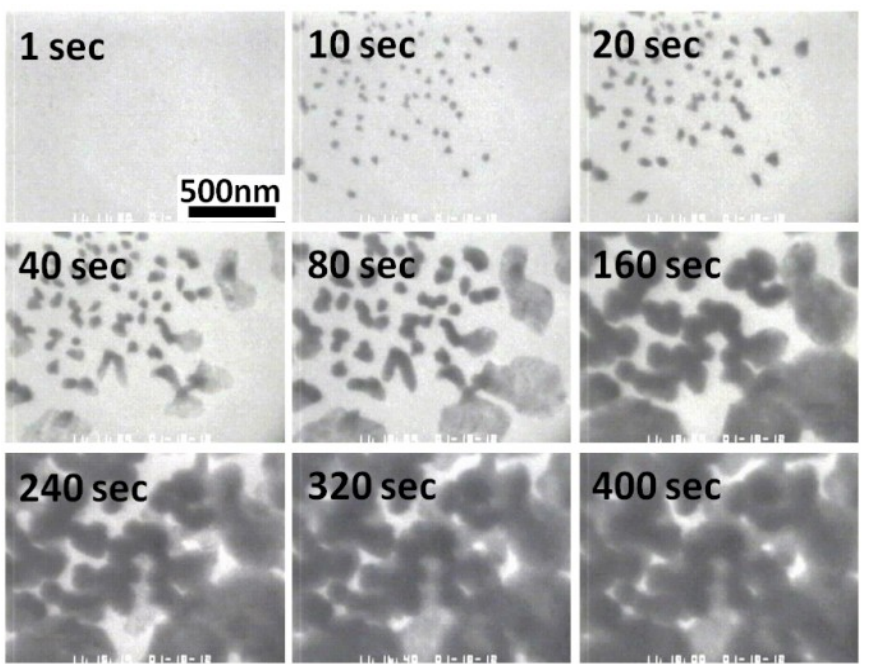

Figure 1. Images extracted from a bright field TEM movie obtained during nucleation and growth of Au crystallites on the liquid cell $\mathrm{SiN}_{\mathrm{x}}$ windows. The liquid layer was several hundred nanometers thick and the beam current was $2.1 \mathrm{nA}$.

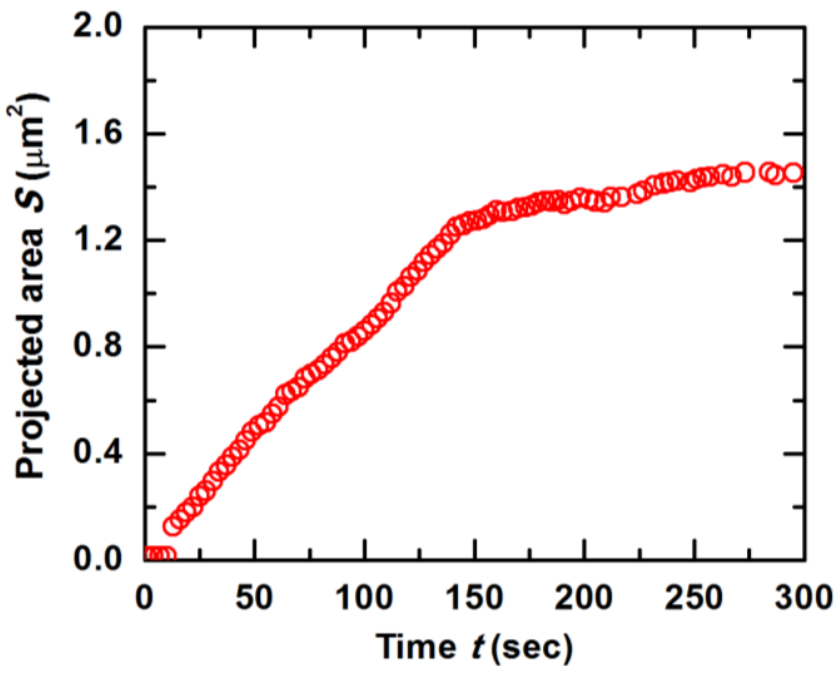

Figure 2. The projected area $S$ of Au crystallites vs. irradiation time $t$. The growth rate $(d S / d t)$ shows distinct regimes. For intermediate times $(10<t<145 \mathrm{sec}), d S / d t$ is constant at 0.0086 $\mu \mathrm{m}^{2} / \mathrm{sec}$. For later times $(145<t<320 \mathrm{sec})$, coalescence occurs and $d S / d t$ decreases to 0.0013 $\mu \mathrm{m}^{2} / \mathrm{sec}$. 